\title{
TRACE FUNCTIONS IN THE RING OF FRACTIONS OF POLYCYCLIC GROUP RINGS
}

\author{
A. I. LICHTMAN
}

Dedicated to the memory of I. N. Herstein

\begin{abstract}
Let $K G$ be the group ring of a polycyclic-by-finite group $G$ over a field $K$ of characteristic zero, $R$ be the Goldie ring of fractions of $K G$, $S$ be an arbitrary subring of $R_{n \times n}$. We prove that the intersection of the commutator subring $[S, S]$ with the center $Z(S)$ is nilpotent. This implies the existence of a nontrivial trace function in $R_{n \times n}$.
\end{abstract}

Let $G$ be a polycyclic-by-finite group, $K$ be a commutative field of characteristic zero. (Throughout this paper the term "field" is used in the sense of "skew field.") It is well known that the group ring $K G$ is semiprime Noetherian and hence has a Goldie ring of fractions which we denote by $R$. Let $S$ be a subring of the matrix ring $R_{n \times n}, Z(S)$ be its center and $[S, S]$ be the $K$-subalgebra of $R_{n \times n}$ generated by all the commutators $[x, y]=x y-y x$, $x, y \in S$. Our first main result is the following theorem which is motivated by R. Snider's article [1].

The intersection

$$
[S, S] \cap Z(S)
$$

is a nilpotent ring (see Theorem 3). (It is known that (1.1) is a subring; the proof of this fact is easy.)

We obtain immediately from Theorem 3 an affirmative answer to the question, posed by R. Snider in [1]: Let $G$ be a poly-Z-group, $K$ be a commutative field of characteristic zero, $D$ be the field of fractions of $K G$. Does

$$
[D, D] \neq D \text { ? }
$$

In particular, does

$$
1 \notin[D, D]
$$

We see thus that the relations (1.2) and (1.3) do hold in $D$. Furthermore, this result implies that there exists a nontrivial trace function $t: D \rightarrow D /[D, D]$, defined by

$$
t(d)=d+[D, D]
$$

Received by the editors September 18, 1989 and, in revised form, January 23, 1990.

1980 Mathematics Subject Classification (1985 Revision). Primary 20C07, 16A27; Secondary 16A08, 16A39.

Partially supported by NSF Grant DMS 8802634. 
and this function can be extended to a function $T: D_{n \times n} \rightarrow D /[D, D]$ by

$$
t\left(d_{i j}\right)=\sum_{i} t\left(d_{i i}\right)
$$

where $\left(d_{i j}\right)$ is an arbitrary matrix from $D_{n \times n}$ (see [1-3]). Snider proved in [1] the relation (1.3) and hence the existence of nontrivial trace functions in the case when $G$ is abelian-by-\{infinite cyclic $\}$. 2):

The proof of Theorem 3 will be based on the following result (see Theorem

Let $K$ be an arbitrary commutative field and $R$ be the ring of fractions of $K G$ and

$$
x_{j} \quad(j=1,2, \ldots, m)
$$

be given nonzero elements of $K G$. Then there exists an ideal $C \subseteq K G$ such that the quotient ring $(K G) / C$ is a finite-dimensional $K$-algebra $K[\widetilde{G}]$, generated by a finite group $\widetilde{G}$ which is the image of $G$ in $(K G) / C$. The homomorphism $\alpha: K G \rightarrow K[\widetilde{G}]$ is extended to a specialization $\theta: R \rightarrow K[\widetilde{G}]$, whose domain $R_{0}$ contains the elements (1.4). Furthermore the elements $\tilde{x}_{j}=\theta\left(x_{j}\right) \quad(j=$ $1,2, \ldots, m)$ are nonzero elements of $K[\widetilde{G}]$.

We will obtain one more result on specializations from $R$ to algebras finitedimensional over their central subfields; this is Theorem 1 and its corollary. Let $H$ be a torsion-free normal subgroup of finite index in $G$ such that $H / H_{1}$ is free abelian, where $H_{1}$ is the Fitting radical of $H$. Then Theorem 1 essentially states that there exists a $G$-invariant ideal $A \subseteq K H_{1}$ and an ideal $B=(A)(K G)$ such that the quotient algebra $(K G) / B \simeq K[\overline{\bar{H}}]$, where the group $\bar{H}$ is abelianby-finite; the images $\bar{x}_{j}(j=1,2, \ldots, m)$ of the elements (1.4) are nonzero in $K[\bar{G}]$ and a given element $x_{j}$ is regular in $R$ iff its image $\bar{x}_{j}$ is regular in $K[\bar{G}]$. Roseblade's Theorem 11.2.9 in [4] implies that the ideal $B$ is localizable in $K G$.

It is worth remarking that Theorems 1 and 2 provide a method for construction of specializations from $R$ into finite-dimensional algebras over the same field $K$; they should be compared with the Reduction Theorem (see [5, Theorem 4.1], [6], or [7, 4.2.1]) which gives specializations into algebras over fields of finite characteristic (see a discussion on this in the book [7, p. 137]).

Throughout this section let $D$ be a field, generated by a polycyclic-by-finite group $G$. Thus, $D$ is the field of fractions of its subring generated by the group $G$; we denote this subring by $T$. Thus, $T=Z[G]$ or $T=Z_{p}[G]$, depending on the characteristic of $D$.

Lemma 1. Let (1.4) be given nonzero elements of $T$. Then there exists an ideal $A \subseteq T$ such that the quotient ring $T / A \simeq \prod_{r \times r}$, where $\Pi$ is a finite field and the images of the elements (1.4) are invertible in $T / A$.

Proof. Wehrfritz proved (see [8] or [7, 4.3.12]) that if $R$ is a finitely generated subring of $D$, then there exists an ideal $C$ of $R$ of finite index with $\bigcap_{n=1}^{\infty} C^{n}=$ 0 ; furthermore, every quotient ring $R / C^{n}(n=1,2, \ldots)$ is finite. We apply this theorem to the subring $S$ of $D$, generated by the elements $x_{j}, x_{j}^{-1}(j=$ 
$1,2, \ldots, m)$ and find an ideal $B \varsubsetneqq S$ such that the ring $\bar{S}=S / B$ is finite. Since the images of the elements $x_{j} \in T(j=1,2, \ldots, m)$ are invertible in the finite ring $\bar{S}$ they must be invertible in the subring $T /(T \cap B)$. We see now that an arbitrary maximal ideal $A \supseteq(T \cap B)$ satisfies the conclusions of the assertion.

Remark. The current proof of Lemma 1 is somewhat shorter than the proof given in the original version of the paper, where Lemma 1 was obtained as one of the corollaries of the Reduction Theorem [5].

Now let $\Pi[G]$ be a domain, generated by a polycyclic-by-finite group $G$ over a finite field $\Pi$. We see that $\Pi[G] \simeq Z_{p}\left[G_{1}\right]$, where $G_{1}$ is the subgroup of units of $\Pi[G]$, generated by $G$ and the multiplicative group of $\Pi$. We see thus that Lemma 1 is true for this case, when $T=\Pi[G]$. We will use it in this form in the proof of Proposition 1 below.

Proposition 1. Let $K$ be an arbitrary commutative field, $G$ be a torsion-free polycyclic group and let (1.4) be given nonzero elements of $K G$. Then there exists a maximal ideal $A \subseteq K G$ such that the quotient algebra $(K G) / A$ is generated over $K$ by a finite group $\bar{G}$, the image of $G$ under the natural homomorphism $(K G) \rightarrow(K G) / A$, and the images of the elements (1.4) in the ring $K[\bar{G}]$ are invertible.

Proof. We reduce first the proof to the case when the field $K$ is finitely generated. Indeed, assume that the theorem is proved for this special case. Let $K_{1}$ be the finitely generated subfield of $K$, such that $K_{1} G$ contains all the elements (1.4) and $A_{1} \subseteq K_{1} G$ be the ideal, which satisfies all the conclusions of the theorem. Since

$$
(K G) /\left(K A_{1}\right) \simeq K \otimes\left(\left(K_{1} G\right) / A_{1}\right),
$$

we obtain an ideal $K A_{1} \subseteq K G$ such that the algebra $(K G) /\left(K A_{1}\right)$ is generated by a finite group and the images of the elements (1.4) are invertible in it. Since images of the elements (1.4) are invertible in the algebra $(K G) /\left(K A_{1}\right)$ they are invertible in every simple homomorphic image of it; this implies easily that an arbitrary maximal ideal $A \subseteq K G$, which contains $K A_{1}$, satisfies the conclusion of the theorem.

We can assume therefore that the field $K$ is finitely generated. Let $K_{0} \subseteq K$ be a finitely generated subring such that $K$ is the field of fractions of $K_{0}$. We have the following representations for the elements (1.4)

$$
x_{j}=\sum_{i} c_{i j} g_{i} \quad\left(c_{i j} \in K ; j=1,2, \ldots, m\right) .
$$

An arbitrary coefficient $c_{i j}$ in (2.1) has a representation

$$
c_{i j}=a_{i j} b_{i j}^{-1} \quad\left(a_{i j}, b_{i j} \in K_{0}\right) .
$$

We can find a maximal ideal $\mathscr{P} \subseteq K_{0}$ which defines a $p$-adic valuation in $K_{0}$ and contains no one of the elements $a_{i j}, b_{i j}$ in (2.2). If $K_{\mathscr{P}}$ is the ring of fractions of $K_{0}$ with respect to $\mathscr{P}$ then all the coefficients $c_{i j}$ in (2.1) belong to $K_{\mathscr{P}}$ and hence

$$
x_{j} \in K_{\mathscr{P}} G \quad(j=1,2, \ldots, m) .
$$

Now consider the natural homomorphism

$$
\varphi: K_{\mathscr{P}} G \rightarrow\left(K_{\mathscr{P}} G\right) /(\mathscr{P}),
$$


where $(\mathscr{P})$ is the ideal of $K_{\mathscr{P}} G$, generated by the ideal $\mathscr{P} \subseteq K_{\mathscr{P}} G$. We observe that the ring $\left(K_{\mathscr{P}} G\right) /(\mathscr{P})$ is isomorphic to the group ring $\Pi G$, where $\Pi \simeq\left(K_{\mathscr{P}}\right) /(\mathscr{P})$ is a finite field and the elements $\varphi\left(x_{j}\right) \quad(j=1,2, \ldots, m)$ are nonzero. Lemma 1 implies that there exists an ideal $B \subseteq \Pi G$ such that $(\Pi G) / B$ is a simple finite ring and the images $\bar{x}_{j}$ of the elements $\varphi\left(x_{j}\right) \quad(j=$ $1,2, \ldots, m)$ are invertible in the ring $(\Pi G) / B$. This together with the homomorphism (2.3) implies that there exists a homomorphism

$$
\psi: K_{\mathscr{P}} G \rightarrow(\Pi G) / B
$$

such that the elements

$$
\bar{x}_{j}=\psi\left(x_{j}\right) \quad(j=1,2, \ldots, m)
$$

are invertible in the ring $(\Pi G) / B$; clearly, $(\Pi G) / B$ is generated over $\Pi$ by the finite group $\bar{G}=\psi(G)$, i.e.,

$$
(\Pi G) / B \simeq \Pi[\bar{G}] .
$$

Now take a minimal left ideal $V$ in the matrix ring $\Pi[\bar{G}]$; this ideal affords a representation $\rho$ of the group $\bar{G}$ and $\rho(\Pi \bar{G}) \simeq \Pi[\bar{G}]$. Let $\widetilde{K}_{0}$ be the $p$-adic completion of $K_{0},(\pi)$ be the maximal ideal of $\widetilde{K}_{0}$. Since $G$ is polycyclic, the group $\bar{G}$ is solvable and Fong-Swan's Theorem implies that there exists a $\widetilde{K}_{0} \bar{G}$-module $\widetilde{V}$, free over $\widetilde{K}_{0}$, such that $\widetilde{V} /(\pi) \widetilde{V} \simeq V$. (In fact, this theorem is proven in $[9,22.1]$ for the case when the group is $p$-solvable and $\widetilde{K}_{0}$ contains a primitive root of degree $(G: 1)$ from 1 but the last condition is unnecessary (see [10]); this can be shown also by a standard argument based on the Galois theory.) If $\lambda$ is the representation afforded by $\widetilde{V}$ and $\lambda\left(\widetilde{K}_{0} \widetilde{G}\right) \simeq R$ then $R /(\pi R) \simeq \Pi[\bar{G}]$; it is important that the ideal $\pi R$ is quasiregular in $R$.

There exists therefore a system of homomorphisms

$$
\widetilde{K}_{0} G \stackrel{\lambda_{1}}{\rightarrow} \widetilde{K}_{0} \bar{G} \stackrel{\lambda}{\rightarrow} R \stackrel{\lambda_{2}}{\rightarrow} \Pi[\bar{G}]
$$

where $\lambda_{1}$ and $\lambda_{2}$ are homomorphisms of $\widetilde{K}_{0}$-algebras.

The homomorphism

$$
\lambda_{2} \lambda \lambda_{1}: \widetilde{K}_{0} G \rightarrow \Pi[\bar{G}]
$$

maps the elements (1.4) into invertible elements $\bar{x}_{j}(j=1,2, \ldots, m)$. Since the kernel of $\lambda_{2}$ is a quasiregular ideal we conclude easily that the images of the elements (1.4) under the homomorphism

$$
\lambda \lambda_{1}: \widetilde{K}_{0} G \rightarrow R
$$

are invertible elements of $R$. Since the field of fractions of $K_{0}$ coincides with $K$ we see that the field of fractions of $\widetilde{K}_{0}$ is isomorphic to the $p$-adic completion $\widetilde{K}$ of $K$; homomorphism (2.7) is extended to a homomorphism of $\widetilde{K}$-algebras

$$
\mu: \widetilde{K} G \rightarrow \widetilde{K} R .
$$

Since the algebra $\widetilde{K} R$ is generated over $\widetilde{K}$ by the finite group $\bar{G}$, we see that the $K$-algebra $\mu(K G)$ is also generated over $K$ by the group $\bar{G}$, i.e.

$$
\mu(K G) \simeq K[\bar{G}] .
$$


The homomorphism (2.8) carries out the elements (1.4) into invertible elements of $\widetilde{K} R$; we obtain therefore that the images of these elements under the homomorphism (2.9) are invertible elements of $K[\bar{G}]$. We found thus a homomorphism

$$
K G \rightarrow(K G) / A \simeq K[\bar{G}]
$$

which maps the elements (1.4) into invertible elements of $K[\bar{G}]$. We can assume, of course, that $K[\bar{G}]$ is simple, i.e. the ideal $A$ is maximal. The proof is complete.

Let $G$ be a polycyclic-by-finite group, $\rho(G)$ be the Fitting radical of $G$. It is not difficult to verify that $G$ contains a torsion-free normal subgroup $H$ of finite index such that the quotient group $H / \rho(H)$ is free abelian; it is more convenient to denote the subgroup $\rho(H)$ by $H_{1}$. We observe that if $A$ is an arbitrary $G$-invariant ideal of $K H_{1}$ then $B=A(K G)$ is an ideal of $K G$ and $(K G) / B \simeq K[\bar{G}]$, where the group $\bar{G}$ is an extension of the normal subgroup $\bar{H}_{1}$ by the group $\bar{G} / \bar{H}_{1} \simeq G / H_{1}$. Thus, the algebra $K[\bar{G}]$ is isomorphic to an appropriate cross product of the algebra $K\left[\bar{H}_{1}\right]$ and the group $G / H_{1}$ and $K[\bar{H}] \simeq K\left[\bar{H}_{1}\right] *\left(H / H_{1}\right)$.

Theorem 1. Let $K$ be an arbitrary commutative field, char $K=p \geq 0$, and assume that nonzero elements (1.4) of $K G$ are given. Then there exists a $G$ invariant ideal $A \subseteq K H_{1}$ and an ideal $B=(A) K G$ such that

(i) The image $\bar{H}_{1}$ of the group $H_{1}$ under the natural homomorphism

$$
\varphi: K G \rightarrow(K G) / B \simeq K[\bar{G}]
$$

is a finite $p^{\prime}$-group and hence the group $\bar{H}$ is finite-by-free abelian. Furthermore, there exists a free abelian normal subgroup $N \subseteq \bar{G}$ of finite index, which is contained in $\bar{H}$ and central in it, and whose elements are linearly independent over $K$; hence $K[N]$ is isomorphic to the group ring $K N$.

(ii) The images

$$
\bar{x}_{j} \quad(j=1,2, \ldots, m)
$$

of the elements (1.4) are nonzero elements of $K[\bar{G}]$. Furthermore, a given element $x_{j}$ in (1.4) is regular in $K G$ if and only if its image $\bar{x}_{j}$ is regular in $K[\bar{G}]$.

(iii) The ideal $B$ is localizable in $K G$.

Proof. Let $g_{1}, g_{2}, \ldots, g_{n}$ be a transversal for $H$ in $G$. The group ring $K H$ contains no zero divisors of $K G$ and we can form the ring $R$ of fractions of $K G$ with respect to the set $(K H) \backslash 0$. If $D$ is the field of fractions of $K H$ then $R \simeq D \otimes_{K H} K G$ and the transversal $g_{1}=1, g_{2}, \ldots, g_{n}$ gives a basis of the left vector space $R$ over $D$.

We can assume without loss of generality that the set (1.4) contains regular elements and these are the first $m_{1}$ elements

$$
x_{1}, x_{2}, \ldots, x_{m_{1}} \text {. }
$$


These elements must be invertible in $R$; this implies easily that there exist nonzero elements $x_{j}^{\prime}\left(j=1,2, \ldots, m_{1}\right)$ in $K G$ such that

$$
\begin{aligned}
& y_{j}=x_{j}^{\prime} x_{j} \in(K H) \backslash 0 \quad\left(j=1,2, \ldots, m_{1}\right), \\
& x_{j}^{\prime} x_{j}=0 \quad\left(j=m_{1}+1, \ldots, m\right) .
\end{aligned}
$$

Now let

$$
\begin{aligned}
x_{j}=\sum_{\alpha=1}^{n} c_{\alpha j} g_{\alpha}, \quad x_{j}^{\prime}=\sum_{\alpha=1}^{n} c_{\alpha j}^{\prime} g_{\alpha} \\
\left(c_{\alpha j}, c_{\alpha j}^{\prime} \in K H ; \alpha=1,2, \ldots, n ; j=1,2, \ldots, m\right)
\end{aligned}
$$

be the representations of the elements $x_{j}, x_{j}^{\prime}(j=1,2, \ldots, m)$. Let

$$
c_{1}, c_{2}, \ldots c_{r}
$$

be all the nonzero coefficients $c_{i j}$ in (3.3). Let $h_{i}(i \in I)$ be a transversal for $H_{1}$ in $H$ and

$$
c_{\beta}=\sum_{i} \lambda_{i \beta} h_{i} \quad\left(\lambda_{i \beta} \in K H_{1} ; \beta=1,2, \ldots, r\right) .
$$

Similarly, we have for the elements $y_{j}$ in (3.2)

$$
y_{j}=\sum_{i} \mu_{i j} h_{i} \quad\left(\mu_{i j} \in K H_{1} ; j=1,2, \ldots, m_{1}\right) .
$$

Apply now Proposition 1 and find a maximal ideal $A \subseteq K H$ such that $(K H) / A$ $\simeq K[\widetilde{H}]$, where $\widetilde{H}$ is a finite group and for all the elements $\lambda_{i \beta}, \mu_{i j}$ from (3.5) and (3.6) the images of the elements

$$
g_{\alpha}^{-1} \lambda_{i \beta} g_{\alpha}, g_{\alpha}^{-1} \mu_{i j} g_{\alpha} \quad(\alpha=1,2, \ldots, n)
$$

are invertible in $K[\widetilde{H}]$. Let

$$
A_{1}=\bigcap_{\alpha=1}^{n} g_{\alpha}^{-1} A g_{\alpha}, \quad A_{2}=A_{1} \cap K H_{1}, \quad B=\left(A_{2}\right) K G .
$$

Clearly, $A_{1}$ is a $G$-invariant ideal of $K H$ and as a result of this $A_{2}$ is a $G$ invariant ideal of $K H_{1}$. Hence $B$ is an ideal in $K G$. We have already pointed out that the quotient ring $(K G) / B \simeq K[\bar{G}]$, where the group $\bar{G}$ is an extension of the group $\bar{H}_{1}$ by the group $\bar{G} / \bar{H}_{1} \simeq G / H_{1}$; the group $G / H_{1}$ is an extension of the free abelian group $H / H_{1}$ by the finite group $G / H$. On the other hand, we obtain from (3.8),

$$
\left(K H_{1}\right) /\left(K H_{1} \cap B\right) \simeq\left(K H_{1}\right) / A_{2} \simeq\left(K H_{1}\right) /\left(K H_{1} \cap A_{1}\right) .
$$

The first relation in (3.8) shows that the image of $K H$ under the natural homomorphism $(K H) \rightarrow(K H) / A_{1}$ is a subdirect sum of the rings $(K H) /\left(g_{\alpha}^{-1} A g_{\alpha}\right)$ $(\alpha=1,2, \ldots, n)$ which are isomorphic to the simple artinian ring $(K H) / A \simeq$ $K[\widetilde{H}]$; a routine argument (see [5, Lemma 2.9]) implies that in fact $(K H) / A_{1}$ is a direct sum of rings isomorphic to $K[\widetilde{H}]$. This, together with the relation (3.9), implies first of all that the group $\bar{H}_{1}$ which is the image of $H_{1}$ under the homomorphism $K G \rightarrow(K G) / B$, is finite. Furthermore, the images of the 
elements (3.7) under the homomorphism $K H \rightarrow(K H) / A$ are invertible. This implies that the elements

$$
\lambda_{i \beta}, \quad \mu_{i j}
$$

become invertible modulo the ideals $g_{\alpha}^{-1} A g_{\alpha}(\alpha=1,2, \ldots, n)$ and hence they are invertible modulo the ideal $A_{1}=\bigcap_{\alpha=1}^{n} g_{\alpha}^{-1} A g_{\alpha}$. Since the elements (3.10) belong to $K H_{1}$ the second and the third relations in (3.8) imply that they are invertible modulo the ideal $B$. We have already observed that the image of $K H$ in $(K G) / B$ is isomorphic to

$$
K[\bar{H}] \simeq K\left[\bar{H}_{1}\right] *\left(H / H_{1}\right) .
$$

Since the group $H / H_{1}$ is free abelian and all the elements

$$
\bar{\lambda}_{i \beta}, \quad \bar{\mu}_{i j} \quad(i=1,2, \ldots, n)
$$

are invertible in $K\left[\bar{H}_{1}\right]$ we conclude easily that the elements

$$
\bar{c}_{\beta}=\sum_{i} \bar{\lambda}_{i \beta} h_{i} \quad(\beta=1,2, \ldots, r)
$$

and

$$
\bar{y}_{j}=\sum_{i} \bar{\mu}_{i j} h_{i} \quad\left(j=1,2, \ldots, n_{1}\right)
$$

are regular in $K[\bar{H}]$. Since $K[\bar{G}]$ is a free $K[\bar{H}]$-module a routine argument shows that these elements are also regular in $K[\bar{G}]$. We obtain from (3.3)

$$
\begin{aligned}
\bar{x}_{j}=\sum_{\alpha=1}^{n} \bar{c}_{\alpha j} g_{\alpha}, \quad \overline{x_{j}^{\prime}}=\sum_{\alpha=1}^{n} \overline{c_{\alpha j}^{\prime}} g_{\alpha} \\
\quad\left(\bar{c}_{\alpha j}, \overline{c_{\alpha j}^{\prime}} \in K[\bar{H}], \alpha=1,2, \ldots, n ; j=1,2, \ldots, m\right) .
\end{aligned}
$$

Since the elements $\left(3.5^{\prime}\right)$ are nonzero we obtain from $\left(3.3^{\prime}\right)$ that $\bar{x}_{j} \neq 0$ $(j=1,2, \ldots, m)$. The relations

$$
\bar{y}_{j}=\overline{x_{j}^{\prime}} \bar{x}_{j} \quad\left(j=1,2, \ldots, m_{1}\right)
$$

imply, via the regularity of the elements $\left(3.6^{\prime}\right)$, that the elements $\bar{x}_{j} \quad(j=$ $\left.1,2, \ldots, m_{1}\right)$ are regular in $K[\bar{G}]$. Similarly, the relations $\bar{x}_{j} \bar{x}_{j}^{\prime}=0 \quad(j=$ $\left.m_{1}+1, \ldots, m\right)$ imply that the elements $\bar{x}_{j}\left(j=m_{1}+1, \ldots, m\right)$ are zero divisors. We completed thus the proof of statement (ii).

To prove statement (iii) we observe that the ideal $B=\left(A_{2}\right) K G$, where $A_{2}$ is an ideal in the group ring of the nilpotent group $H_{1}$. Since $G$ is polycyclic-byfinite Roseblade's Theorem 11.2.9 in [5] implies that $B$ is localizable and (iii) is proved.

We have already shown that $(K H) / A_{1}$ is a direct sum of rings isomorphic to $(K H) / A_{1} \simeq K[\widetilde{H}]$, where $\widetilde{H}$ is a finite group and $A$ is a maximal ideal of $K H$. Hence the ring $(K H) / A_{1}$ is semisimple artinian. Furthermore, we have a homomorphism

$$
K[\bar{H}] \rightarrow(K[\bar{H}]) / \bar{A}_{1} \simeq K[H] / A_{1}
$$

and the second relation (3.9) implies that

$$
\bar{A}_{1} \cap K\left[\bar{H}_{1}\right]=\overline{0} .
$$


We have already shown that the group $\bar{H}_{1}$ is finite. Assume now that char $K=p$ and prove that $p \nmid\left(\left[\bar{H}_{1}\right]: 1\right)$. Indeed, we observe first of all that the group $\bar{H}_{1}$ is nilpotent since $H_{1}$ is. Assume now that $p \mid\left(\bar{H}_{1}: 1\right)$, let $P$ be the Sylow $p$-subgroup of $\bar{H}_{1}$ and let $\bar{H}_{1} \simeq P \times Q$. The elements $h-1 \quad(h \in P)$ generate a nonzero nilpotent ideal in $K\left[\bar{H}_{1}\right]$ because $P$ is a normal subgroup of $\bar{H}_{1}$. Since $K[H] / A_{1}$ is semisimple we obtain from $(3.12)$ that $(h-1) \in \bar{A}_{1}$ $\left(h \in P\right.$ ) which contradicts (3.13). We proved thus that $\bar{H}_{1}$ is a finite $p^{\prime}$-group.

To complete the proof we need the following assertion which is part of Lemma 3.2 in [5].

Lemma 2. Let $K$ be an arbitrary commutative field and $K[U]$ be a ring, generated by a group $U$, which is an extension of a finite group $V$ be a polycyclic-byfinite group $U / V$. Assume also that $K[U] \simeq K[V] *(U / V)$. Then there exists a characteristic poly\{infinite cyclic\} subgroup $F \subseteq U$ of finite index such that the elements of $F$ are linearly independent over $K$ and, hence, $K[F] \simeq K F$.

Proof. Let $F$ be a poly-infinite cyclic characteristic subgroup of finite index in $U$. Then $F \cap V=1$ and it is not difficult to verify that the elements of $F$ are linearly independent over $K[V]$ and hence over $K$.

We complete now the proof of Theorem 1 . Since $\bar{H}_{1}$ is finite, $\bar{H} / \bar{H}_{1}$ is free abelian, and $H$ is finitely generated we conclude that $\bar{H} / Z$ is finite, where $Z$ is the center of $\bar{H}$. The relation (3.11) implies, via Lemma 2, the existence of a characteristic subgroup $F \subseteq \bar{H}$ of finite index such that $K[F] \simeq K F$. Take now $N=F \cap Z$ and statement (iii) follows. The proof is completed.

Let $R$ and $\bar{R}$ be the ring of fractions of $K G$ and $K[\bar{G}]$ correspondingly. The ring $\bar{R}$ is isomorphic to the ring of fractions of $K[\bar{G}]$ with respect to the subring $K N$; since $(\bar{G}: N)$ is finite we conclude easily that $\bar{R}$ has a finite left dimension over the subfield $T=(K N)(K N)^{-1}$ and as a result of it is finitedimensional over a central subfield $Z \subseteq T$. Furthermore, $\bar{R}$ is a homomorphic image of a suitable cross product $T * \bar{G} / N$; when char $K=0$ this cross product is semisimple artinian and so is $\bar{R}$.

If now nonzero elements (1.4) in $R$ are given then

$$
x_{j}=a_{j} b_{j}^{-1} \quad\left(a_{j} \in K G ; b_{j} \in(K G) \backslash 0 ; j=1,2, \ldots, m\right) .
$$

We apply Theorem 1 to the set of elements $a_{j}, b_{j} \in K G(j=1,2, \ldots, n)$ and obtain via well-known facts of the localization theory the following corollary.

Corollary. Let nonzero elements (3.14) in $R$ be given. Then there exists a localizable ideal $B \subseteq K G$ such that the elements (3.14) belong to the subring $S \subseteq R$, obtained by the localization of the ideal $B$, and $S / B S \simeq \bar{R}$, where $\bar{R}$ is the ring of fractions of $K[\bar{G}]$; the ring $\bar{R}$ has a finite dimension over its central subfield $Z$. Clearly, the ideal $B S$ of $S$ is quasiregular,

Let $Q$ be an arbitrary ring. We recall (see Cohn [11] and Passman [12]) that a specialization from $Q$ on ring $\bar{Q}$ is a homomorphism $\alpha: Q_{0} \rightarrow \bar{Q}$ such that $\operatorname{ker} \alpha$ is a quasiregular ideal of $Q_{0} ; Q_{0}$ is the domain of $\alpha$. Theorem 1 thus gives a method for constructing specializations from the $K$-algebra $R$ to algebras finite-dimensional over their central subfields. Another system of specializations to algebras finite-dimensional over $K$ is obtained from the following theorem. 
Theorem 2. Let $R$ be the ring of fractions of $K G$ and (3.14) be given nonzero elements of $R$. Then there exists an ideal $C \subseteq K G$ such that the quotient ring $(K G) / C$ is a finite-dimensional $K$-algebra, generated by a finite group $\widetilde{G}$, which is the image of $G$ in $(K G) / C$. The homomorphism $\alpha: K G \rightarrow K[\widetilde{G}]$ is extended to a specialization $\theta: R \rightarrow K[\widetilde{G}]$, whose domain $R_{0}$ contains the elements (3.14). Furthermore, $\tilde{x}_{j}=\theta\left(x_{j}\right)(j=1,2, \ldots, m)$ are nonzero elements of $K[\widetilde{G}]$.

Proof. Apply first Theorem 1 and its Corollary and obtain a homomorphism

$$
\beta: K G \rightarrow(K G) / B \simeq K[\bar{G}]
$$

such that the elements (3.14) belong to the subring $S \subseteq R$, the domain of the specialization $\pi: R \rightarrow \bar{R}$ which extend $\beta$, and

$$
\bar{x}_{j}=\pi\left(x_{j}\right) \neq 0 \quad(j=1,2, \ldots, m) .
$$

We recall that $\bar{G}$ contains a free abelian normal subgroup $N$ of finite index such that $K[N] \simeq K N$. Let $T$ be the field of fractions of $K N$ and $g_{1}, g_{2}, \ldots, g_{r}$ be a system of elements of $\bar{G}$ which form a basis of the left vector space $\bar{R}$ over $T$. Let

$$
x_{j}=\sum_{i=1}^{r} a_{i j} g_{i} \quad\left(a_{i j} \in T ; j=1,2, \ldots, m\right) .
$$

Let $a_{1}, a_{2}, \ldots, a_{s}$ be all the elements of $K N$ which occur in the numerators and denominators of the nonzero elements $a_{i j}$ in (3.17); clearly, every element $a_{k}(k=1,2, \ldots, s)$ has a finite number of $\bar{G}$-conjugates. Then apply Proposition 1 and find an ideal $A \subseteq K N$ such that the quotient algebra $(K N) / A \simeq K[\tilde{N}]$ where $\widetilde{N}$ is a finite group and

$$
g^{-1} a_{k} g \notin A \quad(k=1,2, \ldots, s ; g \in \bar{G}) .
$$

Let $A_{1}=\bigcap_{g \in \bar{G}} A$ and $\bar{C}=A_{1}(K \bar{G})$. The same argument as in the proof of Theorem 1 shows that $\bar{C}$ contains no one of the elements (3.17) and $K[\bar{G}] / \bar{C} \simeq$ $K[\widetilde{G}]$, where $\widetilde{G}$ is a finite group.

The ideal $\bar{C}$ is localizable in $K[\bar{G}]$; this can be verified in a straightforward way or obtained from Roseblade's theorem in [5]. We see therefore that the homomorphism $\gamma: K[\bar{G}] \rightarrow K[\widetilde{G}]$ is extended to a specialization $\tau: \bar{R} \rightarrow K[\widetilde{G}]$ and

$$
\tilde{x}_{j}=\tau\left(\bar{x}_{j}\right) \neq 0 .
$$

Finally, let $C$ be the inverse image of the ideal $\bar{C}$ in $K G$. Clearly,

$$
(K G) / C \simeq(K[\bar{G}]) / \bar{C} \simeq K[\widetilde{G}] .
$$

Furthermore, the natural homomorphism

$$
\alpha: K G \rightarrow(K G) / C \simeq K[\widetilde{G}]
$$

is a composition of two homomorphisms $\beta$ and $\gamma$, which are extended to specializations $\pi$ and $\tau$ correspondingly. We obtain from this (see [8, Chapter 6] or [11]) that $\alpha$ can be extended to a specialization $\tau \pi=\theta: \bar{R} \rightarrow K[\widetilde{G}]$, 
whose domain contains the elements (3.14). The assertion follows now from (3.16) and (3.18).

4

We will need in the proof of Theorem 3 the following fact:

Lemma 3. Let $D$ be a field, $x$ be a given matrix from $D_{n \times n}$. Assume that $D$ has a system of subrings $T_{i} \quad(i \in I)$ such that $x \in\left(T_{i}\right)_{n \times n}$ for all $i \in I$ and

(1) given any finite set of elements $M \subseteq D$ there is a $T_{i}$ with $M \subseteq T_{i}$,

(2) each $T_{i}$ has an ideal $U_{i} \neq T_{i}$ such that the image of the matrix $x$ in the quotient ring

$\left(T_{i}\right)_{n \times n} /\left(U_{i}\right)_{n \times n} \simeq\left(T_{i} / U_{i}\right)_{n \times n}$ is nilpotent. Then the matrix $x$ is nilpotent. Proof. Assume that $x$ is not nilpotent and hence

$$
x^{n} \neq 0 \text {. }
$$

The powers of $x$ are linearly dependent over $D$; there exists therefore elements $0 \neq d_{j} \in D \quad(j=1,2, \ldots, r)$ such that

$$
\sum_{j=1}^{r} d_{j} x^{n_{j}}=0 \quad\left(1 \leq n_{1}<n_{2}<\cdots<n_{r} \leq n^{2}+1\right) .
$$

Find in the system of subrings $T_{i}(i \in I)$ a subring $T$ and its ideal $U \neq T$ such that $T$ contains all the elements $d_{j}, d_{j}^{-1}(j=1,2, \ldots, r)$, all the nonzero entries of the matrix $x$ (and $x^{n}$ ) and the inverses of these entries. Since all these elements are invertible in $T$ and $U \neq T$, their images in $T / U$ are nonzero. Let $\bar{X}$ denote the image of a subset $X \subseteq T_{n \times n}$ under the homomorphism $(T)_{n \times n} \rightarrow(T / U)_{n \times n}$. We see that the elements $\bar{d}_{j}$ are invertible in $(T / U)_{n \times n}$, the element $\bar{x}$ is nilpotent but

$$
\bar{x}^{n} \neq \overline{0}
$$

and

$$
\sum_{j=1}^{r} \bar{d}_{j} \bar{x}^{n_{j}}=\overline{0}
$$

Now let $k$ be the smallest natural number such that $\bar{x}^{k}=0$. It follows from (4.3) that $k>n$. We multiply $\left(4.2^{\prime}\right)$ on the right by $\bar{x}^{k-n_{1}-1}$ and obtain that $\bar{d}_{1} \bar{x}^{k-1}=0$. Since $\bar{d}_{1}$ is invertible we see that $\bar{x}^{k-1}=0$ which contradicts (4.3). Thus assumption (4.1) leads to a contradiction, i.e., $x$ is nilpotent.

The following fact is known (see [13, Lemma II.5.4]).

Lemma 4. Let $U$ be a finite-dimensional algebra over a field $K$ of characteristic zero, $Z$ be its center. Then the intersection $[U, U] \cap Z$ is a nilpotent ring.

We can now prove our main result.

Theorem 3. Let $G$ be a polycyclic-by-finite group, $K$ be a field of characteristic zero and $R$ be the ring of fractions of $K G$. Let $S$ be a subring of the matrix ring $R_{n \times n}, Z$ be its center. Then the intersection $[S, S] \cap Z$ is a nilpotent ring. Proof. In order to prove Theorem 3 it is enough to prove that the ring $[S, S] \cap Z$ is nil because a nil subring of a matrix ring over the artinian ring $R$ must be nilpotent. 
Let thus $z \in([S, S] \cap Z)$, where $S \subseteq R_{m \times m}$. There exist therefore elements $u_{i}, v_{i} \in S(i=1,2, \ldots, r)$ such that

$$
\sum_{i=1}^{r}\left[u_{i}, v_{i}\right]=z .
$$

Pick in $R$ an arbitrary finite subset which has a form

$$
x_{1}, x_{2}, \ldots, x_{k} ; \quad x_{1}^{-1}, x_{2}^{-1}, \ldots, x_{k}^{-1}
$$

and contains all the nonzero entries of the matrices $u_{i}, v_{i}(i=1,2, \ldots, r)$ (and of $z$ ). Apply Theorem 2 and find a subring $T \subseteq R$ and an ideal $U \subseteq T$ such that elements (4.5) belong to $T$ and $T / U \simeq K[\widetilde{G}]$, where $K[\widetilde{G}]$ is a finitedimensional algebra over $K$. Relation (4.4) implies the following relation in $(T / U)_{m \times m}$ for the images of the elements $u_{i}, v_{i}, z(i=1,2, \ldots, r)$ :

$$
\sum_{i=1}^{r}\left[\bar{u}_{i}, \bar{v}_{i}\right]=\bar{z} .
$$

Since the element $\bar{z}$ commutes with all the elements $\bar{u}_{i}, \bar{v}_{i}(i=1,2, \ldots, r)$, we obtain from Lemma 4 that $\bar{z}$ is nilpotent. Lemma 3 now implies that $z$ is nilpotent which completes the proof of Theorem 3.

Corollary 1. Let the subring $S$ in Theorem 3 be semiprime. Then $[S, S] \cap Z=$ 0 .

Now let $G$ be a residually torsion-free nilpotent group, $K$ be a commutative field. Let

$$
G=N_{1} \supseteq N_{2} \supseteq \cdots
$$

be a series of normal subgroups in $G$ such that every quotient group $G / N_{i}$ $(i=1,2, \ldots)$ is torsion-free nilpotent and $\bigcap_{i=1}^{\infty} N_{i}=1$. It is not difficult to define in $G$ an order such that all the homomorphisms $G \rightarrow G / N_{i}$ are homomorphisms of ordered groups (see [14]). Let $K\langle G\rangle$ be the appropriate Malcev-Neumann power series ring and $\Delta$ be its subfield, generated by the group ring. We will give now a sketch of proof of the following result.

Proposition 2. (i) If char $K=0$ then the conclusion of Theorem 3 is valid for an arbitrary subring $S \subseteq \Delta_{n \times n}$.

(ii) If $K$ has an arbitrary characteristic then

$$
1 \notin[\Delta, \Delta] \text {. }
$$

Proof. Let $\Delta_{i}$ be the field of fractions of the group ring $K\left(G / N_{i}\right)$. The results of [14] imply that for every given $i$ there exists a specialization $\theta_{i}: \Delta \rightarrow \Delta_{i}$, extending the natural homomorphism $G \rightarrow G / N_{i}$ and that for every given elements of $D$,

$$
x_{1}, x_{2}, \ldots, x_{k} ; \quad x_{1}^{-1}, x_{2}^{-1}, \ldots, x_{k}^{-1}
$$

an index $i_{0}$ can be found such that for every $i \geq i_{0}$ these elements belong to the domain $T_{i}$ of the specialization $\theta_{i}$. Since Theorem 3 holds for the subrings of $\left(\Delta_{i}\right)_{n \times n}$ we obtain now easily from Lemma 3 the statement (i). 
We prove now (ii). A routine argument reduces the proof to the case when the group $G$ is finitely generated; we can assume also that the field $K$ is algebraically closed. Assume that $1 \in[\Delta, \Delta]$, i.e. there exist nonzero elements $u_{j}, v_{j} \in \Delta(j=1,2, \ldots, s)$ such that

$$
1=\sum_{j=1}^{s}\left[u_{j}, v_{j}\right]
$$

Apply Proposition 2.8 in [15] and find a specialization $\pi: \Delta \rightarrow K[\widetilde{G}]$ such that $K[\widetilde{G}]$ is a simple algebra generated by a finite $q$-group $\widetilde{G}$ where $q$ is an arbitrary prime number unequal to char $K$ and the domain $T$ of $\pi$ contains all the elements $u_{i}, v_{i}$ from (4.7). The relation (4.7) now yields the following relation in $K[\widetilde{G}]$,

$$
\tilde{1}=\sum_{j=1}^{s}\left[\tilde{u}_{j}, \tilde{v}_{j}\right] .
$$

Since $K[\widetilde{G}]$ is a simple algebra over an algebraically closed field $K$ and $q \neq$ char $K$ we obtain that $K[\widetilde{G}]$ is isomorphic to a matrix algebra of degree $q^{m}$ over $K$. The relation $\left(4.7^{\prime}\right)$ however is impossible in the algebra $K_{q^{m} \times q^{m}}$ since the trace of the right side is zero whereas $T_{r}(\tilde{1})=q^{m} \neq 0$. This completes the proof.

Since free groups and free soluble groups are residually torsion-free nilpotent, we obtain that Propositon 2 is valid for the universal field of fractions of free group rings or for Ore fields of fractions of group rings of free soluble groups.

The truth of (4.6) for a ring of fractions $R$ of a ring $(K G) / P$, where $G$ is a finitely generated nilpotent group, $\operatorname{char} K=0$ and $P$ is a prime ideal of $K G$, was established by M. Lorenz in [16].

\section{ACKNOWLEDGMENT}

The author is grateful to the referee and to B. A. F. Wehrfritz for useful remarks, which helped to avoid some errors and confusion.

\section{REFERENCES}

1. R. L. Snider, The division ring of fractions of a group ring, Lecture Notes in Math., vol. 1029, Springer-Verlag, 1983, pp. 325-339.

2. A. Hattori, Rank element of a projective module, Nagoya J. Math. 15 (1965), 113-120.

3. J. Stallings, Centerless group - an algebraic formulation of Gottlieb's theorem, Topology 4 (1965), 129-134.

4. D. S. Passman, The algebraic structure of group rings, Wiley-Interscience, New York, 1977.

5. A. I. Lichtman, On normal subgroups of the multiplicative group of skew fields generated by a polycyclic-by-finite group, J. Algebra 78 (1982), 548-577.

6. _ On linear groups of a field of fractions of a polycyclic group ring, Israel J. Math. 42 (1982), 318-326.

7. M. Shirvani and B. A. F. Wehrfritz, Skew linear groups, Cambridge Univ. Press, Cambridge, 1986.

8. B. A. F. Wehrfritz, On division rings generated by polycyclic groups, Israel J. Math. 47 (1984), 154-164. 
9. C. Curtis and I. Reiner, Methods of representation theory, vol. I, Wiley-Interscience, New York, 1981.

10. P. Schmid, Lifting modular representations of p-solvable groups, J. Algebra 83 (1983), 461470.

11. P. M. Cohn, Free rings and their relations, Academic Press, New York, 1985.

12. D. S. Passman, Universal fields of fractions for polycyclic group algebras, Glasgow Math. J. 23 (1982), 103-113.

13. N. Jacobson, Lie algebras, Wiley-Interscience, New York, 1962.

14. A. Eisenbud and A. I. Lichtman, On embedding of group rings of residually torsion free nilpotent groups into skew fields, Trans. Amer. Math. Soc. 299 (1987), 373-385.

15. A. I. Lichtman, On PI-subrings of matrix rings over some classes of skew fields, J. Pure Appl. Algebra 52 (1988), 77-89.

16. M. Lorenz, Division algebras generated by finitely generated nilpotent groups, Proc. NATO ASI, Methods in Ring Theory, Reidel, Boston, Mass., 1984, pp. 265-280.

Department of Mathematics, University of Wisconsin-Parkside, Kenosha, Wisconsin 53141 